\title{
Philosophiques
}

Bruce Bégout, La Généalogie de la logique. Husserl, l'antéprédicatif et le catégorial, Paris, Librairie Philosophique J. Vrin, coll. " Bibliothèque d'histoire de la philosophie ", 2000, 379 pages.

\section{Michel Ratté}

Volume 30, numéro 2, automne 2003

URI : https://id.erudit.org/iderudit/008654ar

DOI : https://doi.org/10.7202/008654ar

Aller au sommaire du numéro

Éditeur(s)

Société de philosophie du Québec

ISSN

0316-2923 (imprimé)

1492-1391 (numérique)

Découvrir la revue

Citer ce compte rendu

Ratté, M. (2003). Compte rendu de [Bruce Bégout, La Généalogie de la logique. Husserl, l'antéprédicatif et le catégorial, Paris, Librairie Philosophique J. Vrin, coll. « Bibliothèque d'histoire de la philosophie », 2000, 379 pages.]

Philosophiques, 30(2), 449-453. https://doi.org/10.7202/008654ar d'utilisation que vous pouvez consulter en ligne.

https://apropos.erudit.org/fr/usagers/politique-dutilisation/ 


\title{
Comptes rendus
}

\author{
Bruce Bégout, La Généalogie de la logique. Husserl, l'antéprédicatif et \\ le catégorial, Paris, Librairie Philosophique J. Vrin, coll. «Bibliothèque \\ d'histoire de la philosophie », 2000, 379 pages.
}

Bruce Bégout, cotraducteur français du volume XI des Husserliana paru sous le titre De la synthèse passive. Logique transcendantale et constitutions originaires (Grenoble, Jérôme Million, 1998), nous propose une vaste reconstruction de la problématique husserlienne des synthèses passives en insistant sur son articulation à la question du fondement de la logique transcendantale. L'ouvrage est un contrepoint à celui d'Elmar Holenstein (Phänomenologie der Assoziation. Zu Struktur und Funktion eines Grundprinzips der passiven Genesis bei E. Husserl, La Haye, Nijoff, 1972), que l'auteur a d'ailleurs contribué à faire connaître en français en traduisant le chapitre IX de son livre (voir "L'association en tant que synthèse passive chez Husserl », Philosophie, n 90, juin 1996). C'est dire sa valeur. L'auteur retrace avec assiduité les apories du projet husserlien tranchées trop hâtivement par Holenstein et plusieurs autres. Si cette contribution est hautement spécialisée, Bégout n'en a pas moins le souci de la rendre incontournable pour le public francophone en présentant de manière polémique l'important problème husserlien comme injustement gommé par la phénoménologie française. On sait à quel point un Merleau-Ponty, par exemple, a pu prétendre que la phénoménologie husserlienne avait trouvé dans la problématique de l'intentionnalité pré-réflexive du corps propre un champ de l'expérience passive (sans intervention du moi) qui faisait échec à la prétention de maintenir une valeur fondamentale à la recherche phénoménologique fondée sur l'ego. L'auteur demande alors: "si la genèse passive était la marque d'un débordement décisif de l'expérience phénoménologique hors de l'idéalisme transcendantal, fondé sur la conjonction de la constitution égologique et de la logique, pourquoi Husserl [lui-même] n'en fait-il pas état? Pourquoi n’a-t-il jamais émis le moindre doute concernant l'articulation possible des analyses génétiques de la passivité avec le reste de la phénoménologie transcendantale [...] alors que selon l'interprétation de la phénoménologie française, le développement des différentes intentionnalités passives [...] aurait dû rendre impossible une telle souveraineté de l'ego constituant? Pourquoi, en un mot, l'analyse de la passivité n'a-t-elle pas mené Husserl là où elle a mené Merleau-Ponty, Lévinas ou Henry?» (p. 9). À cette question, l'auteur répond à travers une étude minutieuse de la genèse théorique des problèmes spécifiques qui intéressent la phénoménologie des synthèses passives chez Husserl.

Dans les deux premiers chapitres de la première partie (p. 71-166), l'auteur expose les jalons de la formation du concept husserlien de "synthèse passive ", depuis les traces qu'il en trouve avec Holenstein dans les «synthèses dans le contenu " de la Philosophie de l'arithmétique (1891), dans la structure "horizonale» de la perception et de la temporalité, jusqu'aux "synthèses esthésiques » des Ideen II. Le tout culmine avec l'analyse des recherches spécifiques sur les synthèses passives de contenus sensibles menant Husserl à l'énonciation d'une théorie de l'association originaire: la théorie des théories des synthèses pré-égoïques chez Husserl. 
Dans la deuxième partie du livre (p. 233-346), l'auteur met en lumière l'articulation husserlienne de l'expérience antéprédicative à la question du fondement de la logique dans la mesure où la constitution passive sert précisément chez Husserl à valider la phénoménologie transcendantale «en fondant la logique sur le sol de l'expérience, en élargissant le domaine d'appréhension de l'ego aux dimensions de l'affectivité originaire, de la vie instinctive et de la temporalité primitive du présent vivant» (p. 9).

Les chapitres introductifs (p. 17-68) avertissent de la compréhension spécifique de la passivité chez Husserl: elle n'est évidemment pas pure réceptivité du moi à l'égard de données impressionnelles atomisées, mais "passivité constituante", et se révèle d'emblée dans la co-donation originaire de tout ce qui se donne, en dépendance réciproque et sans intervention du moi. Cela est déjà exemplaire dans la structure arrière-plan/thème de la perception autant que dans la liaison indépassable du maintenant originaire et de la rétention dans la structure du temps vécu. Mais cela se révèle de manière limpide dans les synthèses primordiales de tout contenu sensible, toujours au moins duel et articulé par similarité ou contraste. Cette passivité constituante primaire se différencie aussi d'une passivité dite secondaire, celle qui consiste en la modification permanente de la constitution de sédiments de sens disponibles en vertu du continuum de l'expérience égoïque. Par cette distinction très importante entre une passivité primaire et une passivité secondaire sur laquelle il reviendra (p. 86 et s.), Bégout nous donne la possibilité de voir comment la phénoménologie française, préoccupée par l'historicité du monde de la vie (ce qui est clair chez Merleau-Ponty, en tout cas), aplatit la seconde, c'est-à-dire la sédimentation en arrière-plan de tous les actes thématiques de la conscience - en quelque sorte la Urdoxa - sur la passivité originaire qui se veut plutôt une Urkonstitution.

Une question méthodologique va cependant se poser avec ce projet husserlien : si les synthèses originaires de contenus sensibles sont le fond sur lequel s'érige la constitution transcendantale d'objet, il faut tout de même revoir le sens à accorder au concept de réduction phénoménologique. En effet, pour Husserl, celleci ne prépare pas qu'une analyse régionale de l'expérience, mais est liée structurellement à la méthode d'analyse de tout champ phénoménal. Il s'agit là d'une situation de la phénoménologie déjà trouvée problématique par un Merleau-Ponty par exemple. Mais alors que la critique de celui-ci demeure purement externe - l'affirmation de la primordialité du corps subjectif censé faire éclater la méthode husserlienne au nom d'une vérité immanente au phénomène de la "chair » dont l'articulation au "monde » dépasserait l'ego - l'auteur montre comment, chez Husserl, elle reste absolument interne. Elle passe notamment par l'analyse d'autres formes de passivité que celle de la chair. Pour l'essentiel, le problème de Husserl est que l'opérateur de la variation éidétique qui révèle, lors de l'épochè, un horizon d'évidence et de vérité objectale — l'imagination —, n'est d'aucun secours pour l'élucidation des synthèses de contenus originaires puisque la forme de la phénoménalisation des liaisons originaires n'aboutit pas à des structurations intentionnelles remplissables intuitivement mais s'apparente plus à des renvois pré-égoïques d'indication en indication sans telos propre avant l'activité du moi qui y fait une saisie thématique. De plus, pour Husserl, c'est précisément parce que les contenus originaires s'associent de cette manière qu'ils ouvrent la voie à l'erreur et l'illusion - voir l'analyse husserlienne du phénomène d'affleurement du souvenir sans 
intervention du moi, affleurement qui déclenche une série d'associations s'articulant au présent vivant qui fournit l'occasion d'une saisie thématique risquant autant d'être fautive que rectificatrice au sein de l'activité volontaire de ressouvenir du moi (p. 325 et s.)

L'auteur circonscrit bien le paradoxe de Husserl en demandant comment il est possible de thématiser l'associativité originaire à partir de la méthode de la réduction qui est entièrement orientée par le critère d'apodicticité (p. 41). Le paradoxe n'a évidemment pas échappé à Husserl qui avait déjà mis en œuvre depuis longtemps le projet d'élaborer une méthode alternative - celle de la "phénoméno-logie génétique» par opposition à la "phénoménologie statique» — qui s'intéresse précisément aux synthèses pré-objectales. L'auteur rend compte de manière méticuleuse des difficultés inhérentes à cette nouvelle phénoménologie qui doit surmonter le paradoxe pour toute phénoménologie d'avoir à rendre compte par une explication de ce qui apparaît dans la sphère originaire, alors que c'est précisément le rejet de l'explication qui avait présidé à l'élévation de la description au rang de mode d'expression de la vérité dans le contexte de la réduction. En l'occurrence, non seulement la synthèse passive est une expérience synthétique sans intervention du moi, mais elle est phénoménalement émergente d'une strate de contenus virtuels déjà animés de manière inconsciente - ce qui n'est évidemment pas à la portée d'une phénoménologie descriptive. On n'a qu'à penser au problème du souvenir spontané tout juste mentionné: il suppose un réservoir enfoui de souvenirs vivant en vertu d'une disposition à "résonner» en sympathie (la métaphore est husserlienne). L'auteur discute admirablement de toutes les difficultés et tentatives de sortie de l'impasse chez Husserl, tentatives qui sont d'une richesse indéniable (voir les chapitres 3 et 4 de la première partie portant sur la question de l'affection, c'est-à-dire du mode de phénoménalisation du seuil d'apparition des contenus originaires, et sur le problème de l'inconscient, p. 167-229).

Bégout explore également la difficulté supplémentaire de ce qu'il appelle le «conflit des fondements » (thème sur lequel il conclut, d'ailleurs). Il s'agit en fait de comprendre et d'ordonner le rapport de la constitution originaire sans intervention à la sphère égoïque, problème qui appelle inévitablement celui de l'articulation de la phénoménologie génétique (méthodologiquement fragile) et de la phénoménologie statique. L'enjeu est évidemment de savoir quel est le fondement ultime de l'expérience selon Husserl: la pré-constitution originaire sans intervention du moi ou sa validation significative par l'auteur, qui n'hésite jamais à faire état des circonstances atténuantes - c'est-à-dire de la difficultueuse pensée de Husserl sur la question de la passivité - qui ont permis l'avènement du parti pris français en faveur d'une réduction des problèmes ultimes de la phénoménologie à la phénoménologie de la passivité, n’hésite pas plus à trancher en faveur de Husserl. Le conflit des fondements ne serait pas l'ultime impasse. L'auteur remet en perspective les critiques ricœuriennes de la confusion sémantique entourant le concept de fondement chez Husserl, confusion entre fondement "passif " comme sol sur lequel le transcendantal est construit et fondement comme principe de validation de la construction des idéalités érigée sur ce sol (p. 350 et s.). Il n'y aurait pas confusion mais articulation d'un double fondement où les concepts corollaires de la question du fondement (constitution, donation, etc.) sont spécifiés en fonction de la différence qualitative des fondements. La Urkonstitution serait en fait une forme de flux productif par association originaire proto-objectale qui n'a de plénitude de sens 
possible qu'à travers la thématisation validatrice par le moi : la constitution objectale. Le donné originaire est une donation comme "élan vers ", une donation destinée à être saisie et dont l'être donné fusionne avec son orientation vers le moi. Aux limites de la puissance démonstrative analogico-métaphorique, l'auteur nous dit que cette donation n'est pas à proprement parler une donation, mais une dotation de sens qui doit être validée. Cette proposition fédérative de l'auteur en toute fin de parcours fait écho à quelques trop courtes allusions à une résonance sympathique de la pensée de Husserl avec celle de Maine de Biran sur l'articulation originaire de la passivité et de l'activité dans une dualité primitive (p. 227).

L'ambition positive de l'auteur est sans conteste d'étayer la conviction husserlienne que toutes les formes de synthèses passives, avec leur diversité de constitution — celles de la temporalité originaire, de la corporéité kinesthésique, de la formation homogène du champ perceptuel et des premières configuration sensibles "fournissent le "matériau" primaire sur lequel s'exercent les capacités constitutives du moi» (p. 359). Mais on peut se demander si tous les problèmes qu'il soulève dans sa reconstruction de la théorie husserlienne de la passivité ne sont pas relégués aux oubliettes dans sa manière d'introduire de l'harmonie dans ce qu'il avait peutêtre même déjà euphémisé avec sa locution «conflit des fondements " ? Peut-être faut-il en savoir plus sur la valeur heuristique de l'étude comparative de Maine de Biran et Husserl pour pouvoir trancher.

En tout cas, dans le contexte de l'ouvrage, certaines percées importantes n'ont pas été retenues par cette conclusion. C'est le cas, en l'occurrence, de la question de la phénoménologie de l'inconscient, qui restait non résolue pour l'auteur dans la première partie de son livre. Il défendait alors une position ne banalisant pas l'aporie husserlienne contre les hypothèses visant à l'aplanir tant chez Steinbock et Montavont que chez Holenstein. Dans ce contexte, il en allait simplement de la question de ce que peut être une donation d'activité inconsciente. L'allusion alors faite à Maine de Biran nous laissait sur notre faim devant l'exposé magistral de l'aporie. Le tournant final de l'auteur, qui a consisté à modaliser l'être de la donation en "dotation", n'a heureusement pas réussi à faire oublier la problématique.

Cela dit, il est indéniable que Bégout a réussi à susciter la curiosité à l'égard de Maine de Biran en regard de sa problématique. Et cette curiosité devait nécessairement être insatisfaite de l'opinion trop brièvement exposée de l'auteur sur Michel Henry, qui lui aussi a prétendu trouver dans la philosophie biranienne une source inspirante pour comprendre la passivité (élucidée phénoménologiquement chez lui à travers l'analyse de l'auto-affection) à titre de fondement irréductible de la phénoménalité. Le silence de l'auteur sur Henry tient peut-être au fait qu'il classe d'emblée celui-ci parmi les phénoménologues qui ont décidé de trancher la question $\mathrm{du}$ fondement en faveur du domaine originaire des synthèses passives compris dans un sens husserlien dramatisé dans le but de relativiser définitivement le pouvoir constituant de l'ego (p. 352). Or, Elmar Holenstein, considéré par l'auteur comme un commentateur compétent de Husserl sur la question de la passivité, reconnaît lui-même qu'à rebours de Merleau-Ponty, Michel Henry développe un concept de passivité radicalement différent de celui de Husserl, concept qui se réfère à ce qu'il a nommé la "conscience de soi purement immanente» (E. Holenstein, "L'association en tant que synthèse passive chez Husserl », p. 47). Henry prétend refonder la phénoménologie sur la base d'une radicalisation de la phénoménologie de l'être 
de l'ego, et c'est dans cette optique qu'il recourt à la philosophie biranienne - on est loin de Merleau-Ponty et consorts. Bien sûr, l'auteur n'a pas à prendre le détour henrien. Cependant, on ne peut que le regretter quand on connaît la perspicacité de l'auteur et la minutie de son travail.

MICHEL RATTÉ

Université du Québec à Trois-Rivières

\section{John Leslie, Infinite Minds, Oxford, Oxford University Press, 2001, 234 pages.}

Infinite Minds constitue le quatrième ouvrage de John Leslie, qui fait suite à Value and Existence (1979), Universes (1989) et The End of the World (1996). Infinite Minds présente un contenu très riche et couvre un nombre de sujets particulièrement variés. Parmi ces derniers, on peut citer notamment: l'omniscience, le problème du Mal, l'argument du bon réglage (fine-tuning argument), les effets de sélection observationnels, l'identité des indiscernables, le temps, l'infini, la nature de la conscience.

L'ouvrage se situe clairement dans le domaine de la philosophie spéculative. Et Leslie s'y montre essentiellement préoccupé par des considérations non pas de démonstration rigoureuse, mais de plausibilité et de cohérence. Ainsi, il n'hésite pas à attribuer parfois une probabilité assez faible à certaines affirmations.

Un certain nombre de lecteurs pourront être rebutés dès le début par l'affirmation contraire à l'intuition selon laquelle les galaxies, les planètes, les animaux, mais aussi chacun d'entre nous et les objets qui nous environnent, constituent des structures au sein de pensées divines. On peut penser que c'est une telle affirmation qui a motivé le commentaire placé sur la couverture du livre par un lecteur d'Oxford University Press selon lequel il peut être difficile de croire que l'univers est tel que le décrit l'auteur. Cela a été aussi ma réaction première. Mais si certains lecteurs devaient en tirer une conclusion hâtive, ils manqueraient alors, je pense, ce qui constitue le trésor caché du livre. Car Infinite Minds ressemble à un temple somptueux dont l'accès, toutefois, se trouve dissimulé par un portail d'aspect peu engageant. Ceux qui ne franchiront pas la porte, rebutés par l'aspect de cette dernière, n'auront pas l'occasion de contempler les trésors cachés que contient le livre. Car l'ouvrage apparaît comme présentant une structure et une cohérence profondes, basées sur la mise en cohérence de la conception panthéiste de l'univers de l'auteur avec nos perspectives scientifiques actuelles les plus avancées en matière de cosmologie, de physique ainsi qu'avec les solutions de plusieurs problèmes philosophiques contemporains. Montrer de manière synthétique comment une vision panthéiste du monde peut se montrer compatible avec nos points de vue les plus récents en matière d'univers multiples, de physique et d'informatique quantique, de même qu'avec la théorie de la relativité et les discussions récentes relatives à l'omniscience, le problème du Mal, l'argument du bon réglage, les effets de sélection observationnels, etc., apparaît comme une entreprise à la fois immense et profondément originale.

On observera ici que Leslie est coutumier de ce type de travaux d'envergure. Il suffit pour cela de considérer son œuvre relative à l'argument de l'Apocalypse. Il 